\title{
Exponential Atomic Decomposition in Generalized Weighted Lebesgue Spaces
}

\author{
Nasibova N.P. ${ }^{1}$ \\ ${ }^{1}$ Institute of Mathematics and Mechanics of NAS of Azerbaijan \\ Correspondence: Nasibova N.P., Institute of Mathematics and Mechanics of NAS of Azerbaijan. E-mail: \\ natavan2008@gmail.com
}

Received: October 22, 2014 Accepted: November 3, 2014 Online Published: November 19, 2014

doi:10.5539/jmr.v6n4p137

URL: http://dx.doi.org/10.5539/jmr.v6n4p137

\begin{abstract}
This paper treats the exponential linear phase system which consists of eigenfunctions of the discontinuous differential operator. Frame properties of this system are studied in weighted Lebesgue spaces with the variable order of summability.
\end{abstract}

Keywords: system of exponents, frames, weighted space, variable exponent

\section{Introduction}

Perturbed system of exponents $\left\{e^{i \lambda_{n} t}\right\}_{n \in Z}$ plays an important role in the study of spectral properties of discrete differential operators and in the approximation theory. Apparently, the study of basis properties of these systems dates back to the well-known work of (Paley \& Wiener, 1934). Since then, a lot of research has been made in this field (more details can be found in Sedletskii, 2005; Duffin \& Schaeffer, 1952; Young, 1980; Christensen , 2003; Heil, 2011 ). It should be stressed that similar systems are of great scientific interest in the frame theory as well.

Since recently, there arose a great interest in considering various problems, related to some research fields of mechanics and mathematical physics, in generalized Lebesgue spaces $L_{p_{(\cdot)}}$ with a variable summability exponent $p(\cdot)$ (for more information see Kovacik \& Rakosnik , 1991; Xianling \& Dun , 2001; Kokilashvili \& Paatashvili, 2006; Sharapudinov, 2007; Kokilashvili \& Samko, 2003). Application of Fourier method to the problems for partial differential equations in generalized Sobolev classes requires a good knowledge of approximative properties of perturbed exponential systems in generalized Lebesgue spaces. Approximation-related issues in these spaces have been first studied by Sharapudinov, 2007.

In this work, we consider an exponential linear phase system. The study of frame properties of perturbed exponential systems is closely related to the one of similar properties of perturbed sine and cosine systems. Note that the linear phase sine and cosine systems appear when solving partial differential equations by Fourier method. Basis properties of linear phase trigonometric systems have been studied in (Moiseev, 1984; Moiseev, 1987; Bilalov, 1990; Bilalov, 1999; Bilalov. 2001; Bilalov, 2003; Bilalov, 2004; Moiseev, 1998; Moiseev, 1999). This work is dedicated to the study of frame properties (atomic decomposition, frameness) of the exponential piecewise linear phase system in generalized weighted Lebesgue space.

\section{Needful Information}

We will use the usual notations. $N$-will be a set of all positive integers; $Z$ - will be a set of all integers; $Z_{+}=\{0\} \cup N ; R-$ will be the set of all real numbers; $C-$ will stand for the field of complex numbers; $(-)-$ is the complex conjugate ; $\delta_{n k}-$ is the Kronecker symbol; $\chi_{A}-$ is the characteristic function of the set $A$. Let $p:[-\pi, \pi] \rightarrow[1,+\infty)$ be some Lebesgue-measurable function. By $\mathcal{L}_{0}$ we denote the class of all functions measurable on $[-\pi, \pi]$ with respect to Lebesgue measure. Denote

Let

$$
I_{p}(f) \stackrel{\operatorname{def}}{\equiv} \int_{-\pi}^{\pi}|f(t)|^{p(t)} d t
$$

$$
\mathcal{L} \equiv\left\{f \in \mathcal{L}_{0}: I_{p}(f)<+\infty\right\}
$$


With respect to the usual linear operations of addition and multiplication by a number, $\mathcal{L}$ is a linear space as $p^{+}=\sup _{[-\pi, \pi]} \operatorname{vrai} p(t)<+\infty$. With respect to the norm

$$
\|f\|_{p(\cdot)} \stackrel{\text { def }}{\equiv} \inf \left\{\lambda>0: I_{p}\left(\frac{f}{\lambda}\right) \leq 1\right\},
$$

$\mathcal{L}$ is a Banach space, and we denote it by $L_{p(\cdot)}$. Let

$$
\begin{aligned}
& W L \stackrel{\text { def }}{\equiv}\left\{p: p(-\pi)=p(\pi) ; \exists C>0, \quad \forall t_{1}, t_{2} \in[-\pi, \pi]:\left|t_{1}-t_{2}\right| \leq \frac{1}{2} \Rightarrow\right. \\
& \left.\Rightarrow\left|p\left(t_{1}\right)-p\left(t_{2}\right)\right| \leq \frac{C}{-\ln \left|t_{1}-t_{2}\right|}\right\} .
\end{aligned}
$$

Throughout this paper $q(t)$ will denote the conjugate of a function $p(t): \frac{1}{p(t)}+\frac{1}{q(t)} \equiv 1$. Denote $p^{-}=\inf \underset{[-\pi, \pi]}{\operatorname{vrai}} p(t)$. The following generalized Hölder inequality is true

$$
\int_{-\pi}^{\pi}|f(t) g(t)| d t \leq c\left(p^{-} ; p^{+}\right)\|f\|_{p(\cdot)}\|g\|_{q(\cdot)},
$$

where $c\left(p^{-} ; p^{+}\right)=1+\frac{1}{p^{-}}-\frac{1}{p^{+}}$.

Directly from the definition we get the property which will be used in sequel.

Property A. If $|f(t)| \leq|g(t)|$ a.e. on $(-\pi, \pi)$, then $\|f\|_{p(\cdot)} \leq\|g\|_{p(\cdot)}$.

It is easy to prove

Statement 1. Let $p \in W L, p(t)>0, \forall t \in[-\pi, \pi] ;\left\{\alpha_{i}\right\}_{1}^{m} \subset R$. Function $\rho(t)=\prod_{i=1}^{m}\left|t-\tau_{i}\right|^{\alpha_{i}}$ belongs to the space $L_{p_{(,)}}$, if $\alpha_{i}>-\frac{1}{p\left(\tau_{i}\right)}, \quad \forall i=\overline{1, m} ;$ where $-\pi=\tau_{1}<\tau_{2}<\ldots<\tau_{m}=\pi$.

The following facts play an important role in obtaining the main results.

Property B [Xianling \& Dun, 2001 ] If $p(t): 1<p^{-} \leq p^{+}<+\infty$, then the class $C_{0}^{\infty}(-\pi, \pi)$ (class of finite and indefinitely differentiable functions) is everywhere dense in $L_{p(\cdot)}$.

By $S$ we denote the singular integral

$$
S f=\frac{1}{2 \pi i} \int_{\Gamma} \frac{f(\tau)}{\tau-t} d \tau, \quad t \in \Gamma
$$

where $\Gamma \subset C$ is some piecewise Hölder curve on $C$. Let $\rho:[-\pi, \pi] \rightarrow(0,+\infty)$ be some weight function. Define weight class

$$
L_{p(\cdot), \rho(\cdot)}: L_{p(\cdot), \rho(\cdot)} \stackrel{\operatorname{def}}{\equiv}\left\{f: \rho f \in L_{p(\cdot)}\right\} \quad,
$$

furnished with the norm $\|f\|_{p(\cdot), \rho(\cdot)} \stackrel{d e f}{\equiv}\|\rho f\|_{p(\cdot)}$. The validity of the following statement is established in Kokilashvili \& Samko, 2003.

Statement 2 [Kokilashvili \& Samko, 2003]. Let $p \in W L, 1<p^{-}$. Then, singular operator $S$ is acting boundedly from $L_{p(\cdot), \rho(\cdot)}$ to $L_{p(\cdot), \rho(\cdot)}$, if and only if

$$
-\frac{1}{p\left(\tau_{k}\right)}<\alpha_{k}<\frac{1}{q\left(\tau_{k}\right)}, \quad k=\overline{1, m} .
$$

Let $X$ be some Banach space with a norm $\|\cdot\|_{X}$. Then $X^{*}$ will denote its conjugate with a norm $\|\cdot\|_{X^{*}}$. By $L[M]$ we denote the linear span of the set $M \subset X$, and $\bar{M}$ will stand for the closure of $M$. 


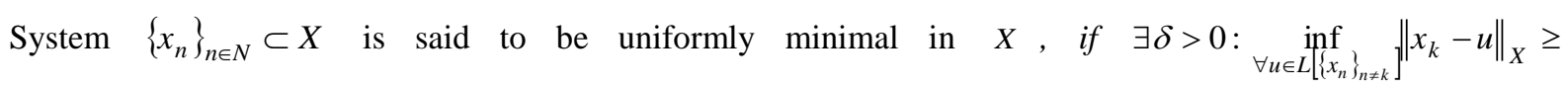
$\delta\left\|x_{k}\right\|_{X}, \forall k \in N$.

System $\left\{x_{n}\right\}_{n \in N} \subset X$ is said to be complete in $X$ if $\bar{L}\left[\left\{x_{n}\right\}_{n \in N}\right]=X$. It is called minimal in $X$ if $x_{k} \notin \widehat{L}\left[\left\{x_{n}\right\}_{n \neq k}\right], \forall k \in N$.

The following criteria of completeness and minimality are available.

Criterion 1. System $\left\{x_{n}\right\}_{n \in N} \subset X$ is complete in $X$, if $f\left(x_{n}\right)=0, \quad \forall n \in N, f \in X^{*} \Rightarrow f=0$.

Criterion 2. System $\left\{x_{n}\right\}_{n \in N} \subset X$ is minimal in $X \Leftrightarrow$ it has a biorthogonal system $\left\{f_{n}\right\}_{n \in N} \subset X^{*}$, i.e. $f_{n}\left(x_{k}\right)=\delta_{n k}, \forall n \cdot k \in N$.

Criterion 3. Complete system $\quad\left\{x_{n}\right\}_{n \in N} \subset X$ is uniformly minimal in $X \Leftrightarrow \sup _{n}\left\|x_{n}\right\|\left\|_{X}\right\| y_{n} \|_{X^{*}}<+\infty$, where $\left\{y_{n}\right\}_{n \in N} \subset X^{*}$ is a system biorthogonal to it.

System $\left\{x_{n}\right\}_{n \in N} \subset X$ is said to be a basis for $X$, iffor $\forall x \in X \quad \exists !\left\{\lambda_{n}\right\}_{n \in N} \subset K: x=\sum_{n=1}^{\infty} \lambda_{n} x_{n}$.

System $\left\{x_{n}\right\}_{n \in N} \subset X$ is said to be a frame if $\forall x \in \widehat{L}\left[\left\{x_{n}\right\}_{n \in N}\right], \exists\left\{\lambda_{n}\right\}_{n \in N} \subset K: x=\sum_{n=1}^{\infty} \lambda_{n} x_{n}$.

If system $\left\{x_{n}\right\}_{n \in N} \subset X$ forms a basis for $X$, then it is uniformly minimal.

We will also need some facts about an atomic decomposition and frames in Banach spaces.

Definition 1. Let $X$ be a Banach space and $\mathscr{K}$ a Banach sequence space indexed by $N$. Let $\left\{f_{k}\right\}_{k \in N} \subset X,\left\{g_{k}\right\}_{k \in N} \subset X^{*}$. Then $\left(\left\{g_{k}\right\}_{k \in N},\left\{f_{k}\right\}_{k \in N}\right)$ is an atomic decomposition of $X \quad$ with respect to $\mathscr{K}$ if:

(i) $\left\{g_{k}(f)\right\}_{k \in N} \in \mathscr{K}, \forall f \in X$;

(ii) $\exists A, B>0: A\|f\|_{X} \leq\left\|\left\{g_{k}(f)\right\}_{k \in N}\right\|_{\mathscr{K}} \leq B\|f\|_{X}, \quad \forall f \in X$;

(iii) $f=\sum_{k=1}^{\infty} g_{k}(f) f_{k}, \forall f \in X$.

The concept of the frame is a generalization of the concept of an atomic decomposition.

Definition 2. Let $X$ be a Banach space and $\mathscr{K}$ a Banach sequence space indexed by $N$. Let $\left\{g_{k}\right\}_{k \in N} \subset X^{*}$ and $S: \mathscr{K} \rightarrow X$ be a bounded operator. Then $\left(\left\{g_{k}\right\}_{k \in N}, S\right)$ is a Banach frame for $X$ with respect to $\mathscr{K}$ if :

(i) $\left\{g_{k}(f)\right\}_{k \in N} \in \mathscr{K}, \forall f \in X$;

(ii) $\exists A, B>0: A\|f\|_{X} \leq\left\|\left\{g_{k}(f)\right\}_{k \in N}\right\|_{\mathscr{K}} \leq B\|f\|_{X}, \quad \forall f \in X$;

(iii) $S\left[\left\{g_{k}(f)\right\}_{k \in N}\right\rfloor=f, \forall f \in X$.

$A$ and $B$ will be called the frame bounds.

It is true the following

Proposition 1. Let $X$ be a Banach space and $\mathscr{K}$ a Banach sequence space indexed by $N$ with canonical basis $\left\{\delta_{n}\right\}_{n \in N}$, where $\delta_{n} \equiv\left\{\delta_{k_{n}}\right\}_{k \in N}$. Let $\left\{g_{k}\right\}_{k \in N} \subset X^{*}$ and $S \in L(\mathscr{K}: X)$. Then the following statements are equivalent:

(i) $\left(\left\{g_{k}\right\}_{k \in N}, S\right)$ is a Banach frame for $X$ with respect to $\mathscr{K}$.

(ii) $\left(\left\{g_{k}\right\}_{k \in N},\left\{S\left(\delta_{k}\right)\right\}_{k \in N}\right)$ is an atomic decomposition of $X$ with respect to $\mathscr{K}$.

\section{Weighted Hardy Classes with a Variable Summability Exponent}

Let $\omega \equiv\{z:|z|<1\}$ be a unit ball on the complex plane, and let $\partial \omega$ be a unit circumference. Denote 


$$
h_{p(\cdot)} \equiv\left\{u: \Delta u=0 \text { in } \omega \text { and }\|u\|_{p(\cdot), \rho}=\sup _{0<r<1}\left\|u\left(r e^{i t}\right)\right\|_{p(\cdot), \rho}<+\infty\right\} .
$$

Lemma 1. Let $p \in W L, p^{-} \geq 1$, and let the weight $\rho(t)$ satisfy condition (1). If $f \in L_{p(\cdot), \rho}$, then $\exists p_{0} \geq 1: f \in L_{p_{0}}$.

In fact, let $f \in L_{p(\cdot), \rho}$. Let us choose $p_{0} \in\left\lfloor 1, p^{-}\right\rfloor$and consider

$$
I_{p_{0}}=\int_{-\pi}^{\pi}|f(t)|^{p_{0}} d t=\int_{-\pi}^{\pi}|f(t)|^{p_{0}} \rho^{\frac{1}{\alpha(t)}} \rho^{-\frac{1}{\alpha(t)}} d t,
$$

where $\alpha(t)=\frac{p(t)}{p_{0}} \geq 1$. Let $\alpha^{*}(t)=\frac{\alpha(t)}{\alpha(t)-1}$. Applying Hölder's inequality, we get

$$
I_{p_{0}} \leq C\left(p^{-}, p^{+}\right)\left\|\left.f\right|^{p_{0}} \rho^{p_{0}}\right\|_{\alpha(\cdot)}\left\|\rho^{-p_{0}}\right\|_{\alpha^{*}(\cdot)} .
$$

It is not difficult to see that

$$
\left\|\left.f\right|^{p_{0}} \rho^{p_{0}}\right\|_{\alpha(\cdot)}=\|f\|_{p(\cdot), \rho} .
$$

It is absolutely clear that the relation $\left\|\rho^{-p_{0}}\right\|_{\alpha^{*}(\cdot)}<+\infty$, holds if and only if the following inequality is fulfilled

Hence we get the condition on the parameter $p_{0}$ :

$$
-p_{0} \alpha^{*}\left(\tau_{k}\right) \alpha_{k}>-1, k=\overline{1, m}
$$

$$
\alpha_{k}<\frac{1}{p_{0}}\left(1-\frac{p_{0}}{p\left(\tau_{k}\right)}\right), k=\overline{1, m} .
$$

As $\lim _{p_{0} \rightarrow 1+0} \frac{1}{p_{0}}\left(1-\frac{p_{0}}{p\left(\tau_{k}\right)}\right)=\frac{1}{q\left(\tau_{k}\right)}, k=\overline{1, m}$, then it follows directly from relations (1) that it is always possible to choose $p_{0} \in\left[1, p^{-}\right]$such that the inequalities (3) are satisfied. As a result, we obtain from (2) that

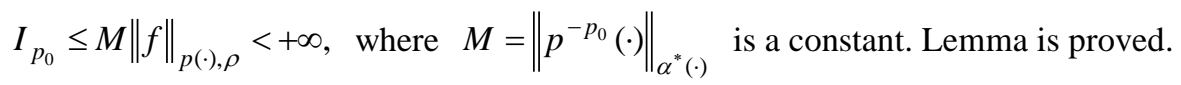

Using this lemma, we prove the following one.

Lemma 2. Let $p \in W L, p^{-} \geq 1$, and let the weight $\rho(t)$ satisfy the condition (1). If $u \in h_{p(\cdot), \rho}$, then $\exists p_{0} \in[1,+\infty]: u \in h_{p_{0}}$.

In fact, let $u \in h_{p(\cdot), \rho}$. Assume

$$
I_{p_{0}}(r)=\int_{-\pi}^{\pi}\left|u_{r}\left(e^{i t}\right)\right|^{p_{0}} d t,
$$

where $u_{r}(\tau)=u(r \tau)$. From Lemma 1 we obtain that $\exists p_{0} \in[1,+\infty]$ :

$$
I_{p_{0}}(r) \leq C\left\|u_{r}(\cdot)\right\|_{p(\cdot), \rho},
$$

where $C$ is a constant (and in further too). Consequently

$$
\sup _{0<r<1}\left(I_{p_{0}}(r)\right) \frac{1}{p_{0}}=\|u\|_{h_{p_{0}}} \leq C\left\|u_{r}(\cdot)\right\|_{p(\cdot), \rho}^{\frac{1}{p_{0}}}<+\infty,
$$

and, as a result $u \in h_{p_{0}}$. Lemma is proved.

The following theorem is true.

Theorem 1. Let $p \in W L, p^{-}>1$, and let the inequalities (1) be satisfied. If $u \in h_{p(\cdot), \rho}$, then $\exists f \in L_{p(\cdot), \rho}$ :

$$
u\left(r e^{i \theta}\right)=\frac{1}{2 \pi} \int_{-\pi}^{\pi} P_{r}(\theta-t) f(t) d t,
$$


where $P_{r}(\alpha)=\frac{1-r^{2}}{1+r^{2}-2 r \cos \alpha}$ is the Poisson kernel. Vice versa, if $f \in L_{p(\cdot), \rho}$, then function $u(\cdot)$, defined by (4), belongs to the class $h_{p(\cdot), \rho}$.

Proof. First, we consider the necessity. Let $u \in h_{p(\cdot), \rho}$. Then, by Lemma $2 \exists p_{0} \in[1,+\infty]: u \in h_{p_{0}}$. Classical results tell us (see e.g. Kusis, 1984) that $\exists f \in L_{p_{0}}$ such that the relation (4) holds. It is known that $u\left(r e^{i \theta}\right) \rightarrow f(\theta)$ as $r \rightarrow 1$ a.e. on $(-\pi, \pi)$ (according to Fatou's lemma Kusis, 1984), and, consequently, $\left|u\left(r e^{i \theta}\right)\right|^{p(\theta)} \rho(\theta) \rightarrow|f(\theta)|^{p(\theta)} \rho(\theta)$ as $r \rightarrow 1$ a.e. on $(-\pi, \pi)$. Then, by the classical Fatou theorem, we obtain that

$$
\int_{-\pi}^{\pi}|f(\theta)|^{p(\theta)} \rho(\theta) d \theta \leq \lim _{r \rightarrow 1} \int_{-\pi}^{\pi}\left|u\left(r e^{i \theta}\right)\right|^{p(\theta)} \rho(\theta) d \theta<+\infty,
$$

i.e. $f \in L_{p(\cdot), \rho}$. Necessity is proved.

Now, let $f \in L_{p(\cdot), \rho}$ and let the relation (4) be true. As the inequalities (1) hold, then it follows from the results of Kokilashvili \& Samko, 2003 that the singular operator (4) with Cauchy kernel is bounded in $L_{p(\cdot), \rho}$. It follows directly that the integral operator (4) with Poisson kernel is uniformly bounded in $L_{p(\cdot), \rho}$ with respect to $\quad r \in(0,1)$, i.e. $\left\|P_{r}(f)\right\|_{p(\cdot), \rho} \leq C\|f\|_{p(\cdot), \rho}$, where $P_{r}(f)=u\left(r e^{i \theta}\right)=\frac{1}{2 \pi} \int_{-\pi}^{\pi} P_{r}(\theta-t) f(t) d t$, and $C$ is a constant independent of $r$ and $f$. Theorem is proved.

Similarly we define the weighted Hardy classes $H_{p(\cdot), \rho}^{ \pm}$. By $H_{p_{0}}^{+}$we denote the usual Hardy class, where $p_{0} \in[1,+\infty)$ is some number. Define $H_{p(\cdot), \rho}^{+} \equiv\left\{f \in H_{1}^{+}: f^{+} \in L_{p(\cdot), \rho}(\partial \omega)\right\}$, where $f^{+}$are non-tangential boundary values on $\partial \omega$ of $f$.

It is absolutely clear that $f$ belongs to the space $H_{p(\cdot), \rho}^{+}$if and only if $\operatorname{Re} f$ and $\operatorname{Im} f$ belong to the space $h_{p(\cdot), \rho}$. Therefore, many of properties of functions from $h_{p(\cdot), \rho}$ stay true for functions from $H_{p(\cdot), \rho}^{+}$. Taking into account the relationship between Poisson kernels $P_{r}(\alpha)$ and Cauchy kernels $K_{z}(t)=\frac{e^{i t}}{e^{i t}-z}$ and using Theorem 1, we easily get the validity of

Theorem 2. Let $p \in W L, p^{-}>1$, and let the inequalities (1) be satisfied. If $F \in H_{p(\cdot), \rho}^{+}$, then $\exists f \in L_{p(\cdot), \rho}$ :

$$
F(z)=\frac{1}{2 \pi} \int_{-\pi}^{\pi} \frac{f(t) d t}{1-z e^{-i t}}=\frac{1}{2 \pi} \int_{-\pi}^{\pi} K_{z}(t) f(t) d t
$$

Vice versa, if $f \in L_{p(\cdot), \rho}$, then the function $F$, defined by (5), belongs to the class $H_{p(\cdot), \rho}^{+}$.

The weighted Hardy class ${ }_{m} H_{p(\cdot), \rho}^{-}$of functions which are analytic in $C \backslash \bar{\omega}(\bar{\omega}=\omega \cup \partial \omega)$ with their orders $m_{0} \leq m$ at infinity is defined similarly to the classical one. Let $f(z)$ be the analytic function in $C \backslash \bar{\omega}$ of finite order $m_{0} \leq m$ at infinity, i.e.

$$
f(z)=f_{1}(z)+f_{2}(z)
$$

where $f_{1}(z)$ is a polynomial of degree $m_{0} \leq m\left(f_{1}(z) \equiv 0 m_{0}<0\right)$, and $f_{2}(z)$ is a regular part of Laurent series expansion of $f(z)$ in the neighborhood of an infinitely remote point. If the function $\varphi(z) \equiv \overline{f_{2}\left(\frac{1}{\bar{z}}\right)}$, belongs to the class $H_{p(\cdot), \rho}^{+}$, then we will say that the function $f(z)$ belongs to the class ${ }_{m} H_{p(\cdot), \rho}^{-}$.

The validity of the following theorem is proved just like in the classical case.

Theorem 3. Let $p \in W L, p^{-}>1$, and let the inequalities (1) be satisfied. If $f \in H_{p(\cdot), \rho}^{+}$, then 


$$
\left\|f\left(r e^{i t}\right)-f^{+}\left(e^{i t}\right)\right\|_{p(\cdot), \rho} \rightarrow 0, r \rightarrow 1-0,
$$

where $f^{+}$are non-tangential boundary values on $\partial \omega$ of $f$.

Just as we have the following

Theorem 4. Let $p \in W L, p^{-}>1$, and let the inequalities (1) be satisfied. If $f \in_{m} H_{p(\cdot), \rho}^{-}$, then

$$
\left\|f\left(r e^{i t}\right)-f^{-}\left(e^{i t}\right)\right\|_{p(\cdot), \rho} \rightarrow 0, r \rightarrow 1+0,
$$

where $f^{-}$are non-tangential boundary values on $\partial \omega$ of $f$ from the outside of $\omega$.

Let us show the validity an analogue of the classical theorem of Smirnov. Assume that $p \in W L, p^{-}>1$ and let the inequality (1) be fulfilled. Let $u \in H_{1}^{+}$and $u^{+} \in L_{p(\cdot), \rho}$, where $u^{+}$be non-tangential boundary value on $\partial \omega$ of $u$. Then it is known that $\exists f \in L_{1}(\partial \omega)$ :

$$
u(z)=\frac{1}{2 \pi i} \int_{\partial \omega} \frac{f(\tau)}{\tau-z} d \tau .
$$

Consequently, $u\left(r e^{i \theta}\right) \rightarrow f\left(e^{i \theta}\right)$ a. e. on $(-\pi, \pi)$ as $r \rightarrow 1-0$. Hence directly follows that $f \in L_{p(\cdot), \rho}$. Then by Theorem 2 we obtain $u^{+} \in H_{p(\cdot), \rho}^{+}$. Thus, the following theorem is true.

Theorem 5. Let $p \in W L, p^{-}>1$, and let the inequalities (1) be satisfied. If $u \in H_{1}^{+}$and $u^{+} \in L_{p(\cdot), \rho}$, then $u^{+} \in H_{p(\cdot), \rho}^{+}$

\section{Bases of exponents in weighted Hardy classes}

Consider the system of exponents $E \equiv\left\{e^{\text {int }}\right\}_{n \in Z}$ and denote $E_{ \pm}^{(k)} \equiv\left\{e^{ \pm \mathrm{int}}\right\}_{n \geq k}$. By $L_{p(\cdot), \rho}^{+}$and ${ }_{m} L_{p(\cdot), \rho}^{-}$we denote the restrictions of classes $H_{p(\cdot), \rho}^{+}$and ${ }_{m} H_{p(\cdot), \rho}^{-}$, respectively, to $\partial \omega$, i.e. $L_{p(\cdot), \rho}^{+}=H_{p(\cdot), \rho}^{+} / \partial \omega ;{ }_{m} L_{p(\cdot), \rho}^{-}={ }_{m} H_{p(\cdot), \rho}^{-} / \partial \omega$. Let's show that if the inequalities (1) hold, then the system $E_{+}^{(0)}$ forms a basis for $L_{p(\cdot), \rho}^{+}$. Take $\forall f \in L_{p(\cdot), \rho}^{+}$. If the inequalities (1) hold, then $L_{p(\cdot), \rho}^{+} \subset L_{1}^{+}$. Then, as is known

$$
\int_{\partial \omega} f(\tau) \tau^{n} d \tau=0, \forall n \in Z_{+} .
$$

More information about this fact can be found in Kusis,1984; Privalov, 1950. If the inequalities (1) hold, then it follows from the results in Danilyuk, 1975 that the system of exponents $E$ forms a basis for $L_{p(\cdot), \rho}$. Taking into account (6), we obtain that $f$ can be expanded in a series in $L_{p(\cdot), \rho}$ of the following form

$$
f\left(e^{\mathrm{int}}\right)=\sum_{n=0}^{\infty} f_{n} e^{\mathrm{int}},
$$

where $f_{n}$ are the biorthogonal coefficients of $f$ with respect to the system $E$. It is absolutely clear that such an expansion is unique. Consequently, the system $E_{+}^{(0)}$ forms a basis for $L_{p(\cdot), \rho}^{+}$. Similarly it can be proved that if the inequalities (1) hold, then the system $E_{-}^{(m)}$ forms a basis for ${ }_{m} L_{p(\cdot), \rho}^{-}$. Thus, the following theorem is true.

Theorem 6. Let $p \in W L, p^{-}>1$, and let the inequalities (1) be satisfied. Then the system $E_{+}^{(0)}\left(E_{-}^{(m)}\right)$ forms a basis for $L_{p(\cdot), \rho}^{+}\left({ }_{m} L_{p(\cdot), \rho}^{-}\right), 1<p<+\infty$.

\section{Riemann Boundary Value Problem for $H_{p(\cdot), \rho}^{ \pm}$Classes}

Consider the following Riemann problem in $H_{p(\cdot), \rho}^{+} \times{ }_{m} H_{p(\cdot), \rho}^{-}$classes

$$
F^{+}(\tau)-G(\tau) F^{-}(\tau)=f(\tau), \tau \in \partial \omega,
$$

where $f \in L_{p(\cdot), \rho}$ is some function. By the solution of problem (7) we mean a pair of analytic functions 
$\left(F^{+}(z) ; F^{-}(z)\right) \in H_{p(\cdot), \rho}^{+} \times_{m} H_{p(\cdot), \rho}^{-}$, boundary values of which satisfy the relation (7) almost everywhere. Introduce the following functions $X_{i}^{ \pm}(z)$, which are analytic inside (with the + sign) and outside (with the sign) the unit circle, respectively:

where $\theta(t) \equiv \arg G\left(e^{i t}\right)$. Define

$$
\begin{gathered}
X_{1}^{ \pm}(z) \equiv \exp \left\{ \pm \frac{1}{4 \pi} \int_{-\pi}^{\pi} \ln \mid G\left(e^{i t}\right) \frac{e^{i t}+z}{e^{i t}-z} d t\right\}, \\
X_{2}^{ \pm}(z) \equiv \exp \left\{ \pm \frac{1}{4 \pi} \int_{-\pi}^{\pi} \theta(t) \frac{e^{i t}+z}{e^{i t}-z} d t\right\},
\end{gathered}
$$

Sokhotski-Plemelj formulas yield

$$
Z_{i}(z) \equiv\left\{\begin{array}{l}
X_{i}^{+}(z),|z|<1, \\
{\left[X_{i}^{-}(z)\right]^{-1},|z|>1, \quad i=1,2 .}
\end{array}\right.
$$

$$
\left|G\left(e^{i t}\right)\right|=\frac{Z_{1}^{+}\left(e^{i t}\right)}{Z_{1}^{-}\left(e^{i t}\right)}, e^{i \theta(t)}=\frac{Z_{2}^{+}\left(e^{i t}\right)}{Z_{2}^{-}\left(e^{i t}\right)} .
$$

Assume $Z^{ \pm}(z) \equiv Z_{1}^{ \pm}(z) Z_{2}^{ \pm}(z)$. We have

$$
Z^{+}(\tau)-G(\tau) Z^{-}(\tau)=0, \quad \tau \in \partial \omega
$$

Introduce the piecewise analytic function

$$
Z(z) \equiv\left\{\begin{array}{l}
Z^{+}(z),|z|<1, \\
Z^{-}(z),|z|>1 .
\end{array}\right.
$$

Following the classics, we call function $Z(z)$ the canonical solution of the problem (7). Substituting the (8) expression for $G(\tau)$ in (7), we obtain

$$
\frac{F^{+}(\tau)}{Z^{+}(\tau)}=\frac{F^{-}(\tau)}{Z^{-}(\tau)}, \quad \tau \in \partial \omega
$$

Let $\Phi^{ \pm}(z) \equiv \frac{F^{ \pm}(z)}{Z^{ \pm}(z)}$, and define the piecewise analytic function

$$
\Phi(z) \equiv\left\{\begin{array}{l}
\Phi^{+}(z),|z|<1, \\
\Phi^{-}(z),|z|>1 .
\end{array}\right.
$$

It is not difficult to see that the function $Z(z)$ has neither poles nor zeros for $z \notin \partial \omega$. Therefore, functions $\Phi(z)$ and $F(z)$ have the same order at infinity. The results of Danilyuk, 1975 imply directly that the function $\Phi(z)$ belongs to the Hardy class $H_{\delta}^{ \pm}$for sufficiently small values of $\delta>0$. Let us show that $\Phi(z) \in H_{1}^{ \pm}$. To do so, it suffices to prove that $\Phi^{ \pm}(\tau) \in L_{1}(\partial \omega)$, because the rest will immediately follow from the Smirnov theorem (Danilyuk, 1975) .

We will suppose that the coefficient $G(\tau)$ satisfies the following conditions:

$$
\text { 1) } G^{ \pm} \in L_{\infty}(\partial \omega)
$$

2) $\theta(t) \equiv \arg \left(e^{i t}\right)$ is a piecewise Hölder function on $[-\pi, \pi]$.

Let $\left\{s_{k}\right\}_{1}^{r}:-\pi<s_{1}<\ldots s_{r}<\pi$ be the points of discontinuity of the function $\theta(t)$ and

$$
\left\{h_{k}\right\}_{1}^{r}: h_{k}=\theta\left(s_{k}+0\right)-\theta\left(s_{k}-0\right), k=\overline{1, r} ;
$$

be the corresponding jumps of $\theta(t)$ at these points. Denote

Let

$$
h_{0}=\theta(-\pi)-\theta(\pi) ; h_{0}^{(0)}=\theta_{0}(\pi)-\theta_{0}(-\pi) .
$$




$$
u_{0}(t) \equiv\left\{\sin \left|\frac{t-\pi}{2}\right|\right\}^{-\frac{h_{0}^{(0)}}{2 \pi}} \exp \left\{-\frac{1}{4 \pi} \int_{-\pi}^{\pi} \theta_{0}(\tau) \operatorname{ctg} \frac{t-\tau}{2} d \tau\right\}
$$

Assume

$$
u(t)=\prod_{k=0}^{r}\left\{\sin \left|\frac{t-s_{k}}{2}\right|\right\}^{\frac{h_{k}}{2 \pi}}, \text { where } s_{0}=\pi .
$$

As is known (see Danilyuk, 1975 ), the boundary values $\left|Z^{-}(\tau)\right|$ are expressed by the formula

$$
\mid Z_{2}^{-}\left(e^{i t}\right)=u_{0}(t)[u(t)]^{-1}\left\{\sin \left|\frac{t-\pi}{2}\right|\right\}^{-\frac{h_{0}}{2 \pi}},
$$

i.e.

$$
\left|Z_{2}^{-}\left(e^{i t}\right)\right|=u_{0}(t) \prod_{k=0}^{r}\left|\sin \frac{t-s_{k}}{2}\right|^{-\frac{h_{k}}{2 \pi}} .
$$

It follows directly from the Sokhotski-Plemelj formula that

$$
\sup _{(-\pi, \pi)} \operatorname{rai}\left\{\mid Z_{1}^{-}\left(e^{i t}\right)^{ \pm 1}\right\}<+\infty \text {. }
$$

Thus, the following representation is true for $\mid Z^{-}\left(e^{i t}\right)^{-1}$ :

$$
\left.\left|Z^{-}\left(e^{i t}\right)^{-1}=\right| Z_{1}^{-}\left(e^{i t}\right)\right|^{-1}\left|u_{0}(t)\right|^{-1} \prod_{k=0}^{r}\left|\sin \frac{t-s_{k}}{2}\right|^{\frac{h_{k}}{2 \pi}} .
$$

By the definition of solution, we have $F^{-}(z) \in H_{p(\cdot), \rho}^{-}$. Consequently, $F^{-}(\tau) \in L_{p(\cdot), \rho}(\partial \omega)$. Therefore, if $\left|Z^{-}(\tau)\right|^{-1} \in L_{q(\cdot), \rho^{-1}}(\partial \omega)$, then we obtain directly from the Hölder inequality that $\Phi^{-}(\tau) \in L_{1}(\partial \omega)$.

We will need the following easy-to-prove lemma that follows directly from Statement 1.

Lemma 3. Let $p \in C[-\pi, \pi]$ and $p(t)>0, \forall t \in[-\pi, \pi]$. Then the function $\xi(t)=|t-c|^{\alpha}$ belongs to $L_{p(\cdot), \rho}$ if $\alpha>-\frac{1}{p(c)}$ for $c \neq \tau_{k}, \forall k=\overline{1, m}$, and $\alpha+\alpha_{k_{0}}>-\frac{1}{p(c)}$ for $c=\tau_{k_{0}}$.

Represent the product $\left|Z^{-} \rho\right|^{-1}$ in the following form

$$
\left|Z^{-} \rho\right|^{-1}=\left|Z_{1}^{-}\right|^{-1}\left|u_{0}\right|^{-1} \prod_{k=0}^{l}\left|t-t_{k}\right|^{\beta_{k}},
$$

where $\left\{t_{k}\right\}_{k=0}^{l} \equiv\left\{\arg \tau_{k}\right\}_{k=1}^{m} \cup\left\{s_{k}\right\}_{k=0}^{r}$, and $\beta_{k}$ is defined by the relation

$$
\beta_{k}=\sum_{i=1}^{m} \alpha_{i} \chi_{\left\{t_{k}\right\}}\left(\arg \tau_{i}\right)+\frac{1}{2 \pi} \sum_{i=0}^{r} h_{i} \chi_{\left\{t_{k}\right\}}\left(s_{i}\right), k=\overline{0, l} .
$$

Taking into account Lemma 3, we obtain that if the inequalities

$$
\beta_{k}>-\frac{1}{q\left(t_{k}\right)}, k=\overline{0, r},
$$

are true, then the product $\left|Z^{-} \rho\right|^{-1}$ belongs to the space $L_{q(\cdot)}$, i.e. $\left|Z^{-}\right|^{-1} \in L_{q(\cdot), \rho^{-1}}$. So, if the inequalities (11) are true, then the function $\Phi(z)$ belongs to classes $H_{1}^{ \pm}$. Consequently, according to the results of Danilyuk, 1975, $\Phi(z)$ is a polynomial $P_{m_{0}}(z)$ of order $m_{0} \leq m$. Thus, $F^{-}(z)=P_{m_{0}}(z) Z^{-}(z)$. Let's find out under which conditions the function $F^{-}(z)$ belongs to the space $H_{p(\cdot), \rho}^{-}$. We have 


$$
\left|Z^{-} \rho\right|=\left|Z_{1}\right|\left|u_{0}\right| \prod_{k=0}^{l}\left|t-t_{k}\right|^{-\beta_{k}} .
$$

Consequently, if the inequalities

$$
\beta_{k}<\frac{1}{p\left(t_{k}\right)}, k=\overline{0, r} \quad,
$$

are true, then it is clear that $F^{-}(\tau) \in L_{p(\cdot), \rho}$, and hence $F^{-} \in{ }_{m} H_{p(\cdot), \rho}^{-}$. So, if the inequalities

$$
-\frac{1}{q\left(t_{k}\right)}<\beta_{k}<\frac{1}{p\left(t_{k}\right)}, k=\overline{0, r}
$$

are true, then the general solution of the homogenous problem

$$
F_{0}^{+}(\tau)=G_{1}(\tau) F_{0}^{-}(\tau), \quad \tau \in \partial \omega
$$

in classes $H_{p(\cdot), \rho}^{+} \times{ }_{m} H_{p(\cdot), \rho}^{-}$can be represented as $F_{0}(z)=P_{m_{0}}(z) Z(z)$, where $P_{m_{0}}(z)$ is an arbitrary polynomial of order $m_{0} \leq m$.

Now let's consider the non-homogenous problem (7). Take $\forall f \in L_{p(\cdot), \rho}$ and suppose

$$
F_{1}(z)=\frac{Z(z)}{2 \pi} \int_{-\pi}^{\pi} K_{z}(t) Z^{+}\left(e^{i t}\right) f(t) d t .
$$

The Sokhotski-Plemelj formulas imply that the boundary values $F_{1}^{ \pm}$satisfy a.e. the equality (7). Moreover, it follows from Statement 2 that $\left(F_{1}^{+}(z) ; F_{1}^{-}(z)\right) \in H_{p(\cdot), \rho}^{+} \times_{-1} H_{p(\cdot), \rho}^{-}$. As a result, we get the validity of the following theorem.

Theorem 7. Let $\left\{\beta_{k}\right\}_{1}^{r}$ be defined by (10) and the inequalities (1), (12) be satisfied. Then the general solution of the Riemann problem (7) in classes $H_{p(\cdot), \rho}^{+} \times_{m} H_{p(\cdot), \rho}^{-}$can be represented in the following form

$$
F(z)=P_{m_{0}}(z) Z(z)+F_{1}(z),
$$

where $Z(\cdot)$ is the canonical solution of homogenous problem, $F_{1}(\cdot)$ is the particular solution of non-homogenous problem (7) defined by (13), and $P_{m_{0}}(\cdot)$ is a polynomial of order $m_{0} \leq m$.

\section{Atomic Decomposition}

Consider the following exponential linear phase system

$$
\mathrm{E} \equiv\left\{\mathrm{E}_{n}(t)\right\}_{n \in Z} \equiv\left\{e^{i(n+s i g n n) t}\right\}_{n \in Z},
$$

where $\alpha \in C$ is a complex parameter. To explore the decomposition with regard to the system (14), we will follow the scheme of Bilalov \& Guseynov, 2012. Consider the conjugate problem

$$
F^{+}\left(e^{i t}\right)-G\left(e^{i t}\right) F^{-}\left(e^{i t}\right)=e^{-i \alpha t} f(t), \quad t \in[-\pi, \pi],
$$

where $G\left(e^{i t}\right)=e^{-2 i \alpha t}$. The particular solution $F_{0}(z)$ of the problem (15) has the following form

$$
\begin{aligned}
& F_{0}^{+}(z)=\frac{(1+z)^{-2 \alpha}}{2 \pi} \int_{-\pi}^{\pi} \frac{e^{i \alpha t} f(t) d t}{\left(1+e^{i t}\right)^{-2 \alpha}\left(1-z e^{-i t}\right)}, \\
& F_{0}^{-}(z)=\frac{\left(1+z^{-1}\right)^{-2 \alpha}}{2 \pi} \int_{-\pi}^{\pi} \frac{e^{-i \alpha t} f(t) d t}{\left(1+e^{i t}\right)^{-2 \alpha}\left(1-z e^{-i t}\right)} .
\end{aligned}
$$

Proceeding as in the previous section, we get $\theta(t)=-\operatorname{Re} \alpha t, \quad r=0, \quad h_{0}=\theta(-\pi)-\theta(\pi)=4 \operatorname{Re} \alpha \pi$. Consequently, $\beta_{0}=\alpha_{m}+2 \operatorname{Re} \alpha, \beta_{k}=\alpha_{k}, \forall k \neq 0$. Then from Theorem 7 we obtain that if the inequalities

$$
\begin{gathered}
-\frac{1}{q\left(\tau_{k}\right)}<\alpha_{k}<\frac{1}{p\left(\tau_{k}\right)}, \quad k=\overline{1, m}, \\
\frac{1}{q(\pi)}<\alpha_{m}+2 \operatorname{Re} \alpha<\frac{1}{p(\pi)}
\end{gathered}
$$

are true, then the conjugate problem (15) has a unique solution $\left(F_{0}^{+}(z) ; F_{0}^{-}(z)\right)$ in classes $H_{p(\cdot), \rho}^{ \pm}$when $F^{-}(\infty)=0$. Also, it can be proved (in the same way as in Bilalov \& Guseynov, 2012; Bilalov \& Guseinov, 
February, 2011) that, with the inequalities (16) and (17) fulfilled, the system (14) forms a basis for $L_{p(\cdot), \rho}$. Thus, the following theorem is true.

Theorem 8. Let $p \in W L, p^{-}>1$, and the inequalities (16),(17) be fulfilled. Then the exponential system (14) forms a basis for the generalized weighted Lebesgue space $L_{p(\cdot), \rho(\cdot)}$.

\section{Atomic Decomposition and Frameness}

Denote by $\mathscr{K}(E)$ the space of coefficients of the system (14). It is known that the canonical system $\left\{\delta_{n}\right\}_{n \in N}$ forms a basis for в $\mathscr{K}(E)$ (see, e.g. Mamedova, 2012; Sadigova \& Mamedova,2013), where $\delta_{n} \equiv\left\{\delta_{n k}\right\}_{k \in N}$. Let $K: \mathscr{K}(E) \rightarrow L_{p(\cdot), \rho}$ be a coefficient operator

$$
K \vec{\lambda}=\sum_{n=-\infty}^{+\infty} \lambda_{n} E_{n}, \quad \vec{\lambda} \equiv\left\{\lambda_{n}\right\}_{n \in Z} \in \mathscr{K}(E) .
$$

$K$ is an isomorphism between $\mathscr{K}(E)$ and $L_{p(\cdot), \rho}$, because the system (14) forms a basis for $L_{p(\cdot), \rho}$ and, moreover, $K \delta_{n}=E_{n}, \forall n \in Z$. Thus,

$$
A\|f\|_{p(\cdot), \rho} \leq\left\|\left\{f_{n}\right\}_{n \in Z}\right\|_{\mathscr{H}(E)} \leq B\|f\|_{p(\cdot), \rho}, \quad \forall f \in L_{p(\cdot), \rho},
$$

where $\left\{f_{n}\right\}_{n \in Z} \equiv K^{-1} f$, and $A, B>0$ are the constants. Let $\mathrm{E}^{*} \equiv\left\{\mathrm{E}_{n}^{*}(t)\right\}_{n \in Z} \subset L_{q(\cdot), \rho}$ be the system conjugate to $E$. Then, by definition (see, e.g. Christensen, 2003), the pair $\left\{E^{*} ; E\right\}$ is an atomic decomposition of $L_{p(\cdot), \rho}$ with regard to $\mathscr{K}(E)$. Moreover, the pair $\left\{E^{*} ; K\right\}$ forms a Banach frame for $L_{p(\cdot), \rho}$ with regard to $\mathscr{K}(E)$.

Suppose that $K_{d}$ is some $B$-space of the sequences of scalars furnished with the norm $\|\cdot\|_{d}$. Let the pair $\left\{G^{*} ; S\right\}$ form a frame for $L_{p(\cdot), \rho}$, where $G^{*} \equiv\left\{g_{n}(t)\right\}_{n \in N} \subset L_{q(\cdot), \rho}$ is some system and $S \in L\left(K_{d} ; L_{p(\cdot), \rho}\right)$ is a frame operator. It is not difficult to see that $S$ performs isomorphism between $\mathscr{K}_{d}^{S}$ and $L_{p(\cdot), \rho}$, where $\mathscr{K}_{d}^{S} \equiv \operatorname{Im} S^{-1}$. Consequently, $S^{-1} \circ K$ performs isomorphism between $\mathscr{K}(E)$ and $\mathscr{K}_{d}^{S}$, i.e. $\mathscr{K}(E)$ and $\mathscr{K}_{d}^{S}$ are isomorphic. Without loss of generality, we will assume that the operator $S$ is defined on $\mathscr{K}_{d}^{S}$, i.e. $S \in L\left(\mathscr{K}_{d}^{S} ; L_{p(\cdot), \rho}\right)$.

Conversely, suppose that the $B$-space of the sequences of scalars $X_{d}$ is isomorphic to $\mathscr{K}(E)$ and $T: X_{d} \leftrightarrow \mathscr{K}(E)$ is the corresponding isomorphism. Assume $S=K \circ T$. It is clear that $S \in L\left(X_{d} ; L_{p(\cdot), \rho}\right)$ is an isomorphism. We have

$$
f=S S^{-1} f=S T^{-1}\left(K^{-1} f\right)=S T^{-1}\left(\left\{E_{n}^{*}(f)\right\}_{n \in Z}\right)=S\left(\left\{g_{n}(f)\right\}_{n \in Z}\right),
$$

where $\left\{g_{n}(f)\right\}_{n \in Z}=T^{-1}\left(\left\{E_{n}^{*}(f)\right\}_{n \in Z}\right) \in X_{d}$, and $E_{n}^{*}(f)$ is the value of functional $E_{n}^{*} \in L_{q(\cdot), \rho}$ at the element $f \in L_{p(\cdot), \rho}:$

$$
E_{n}^{*}(f)=\int_{-\pi}^{\pi} f(t) \overline{E_{n}^{*}(t)} \rho(t) d t .
$$

It is not difficult to see that $g_{n}(f)=T^{-1}\left(\widetilde{E}_{n}^{*}(f)\right)$, where $\tilde{E}_{n}^{*}(f)=\left\{\delta_{n k} E_{k}^{*}(f)\right\}_{k \in Z}, \forall n \in Z$. It hence follows that $\left\{g_{n}\right\}_{n \in Z} \subset L_{q(\cdot), \rho}$, i.e. $g_{n} \in\left(L_{p(\cdot), \rho}\right)^{*}, \forall n \in Z$. We have

$$
m\|f\|_{p(\cdot), \rho} \leq\left\|\left\{g_{n}(f)_{n \in Z}\right\}\right\|_{X_{d}} \leq M\|f\|_{p(\cdot), \rho}, \forall f \in L_{p(\cdot), \rho},
$$

where $\|\cdot\|_{X_{d}}$ is the norm on $X_{d}$, and $m ; M>0$ are the constants. As a result, we obtain that the pair $\left(\left\{g_{n}\right\}_{n \in Z} ; S\right)$ forms a frame for $L_{p(\cdot), \rho}$ with regard to $X_{d}$.

So, let's consider the general case with regard to the system (14). Assume

$$
P_{\pi} \equiv\left\{\frac{1}{2}\left(\frac{1}{p(\pi)}-\alpha_{m}+k\right): \forall k \in Z\right\} .
$$

Let $\operatorname{Re} \alpha \notin P_{\pi}$. Then $\exists k_{0} \in Z$ : 
Consider the following system

$$
-\frac{1}{q(\pi)}<\alpha_{m}+2 \operatorname{Re} \alpha+k_{0}<\frac{1}{p(\pi)} .
$$

$$
\mathrm{E}_{k_{0}} \equiv\left\{e^{i \alpha_{k_{0}} t} e^{\mathrm{int}} ; e^{-i \alpha t} e^{-i k t}\right\}_{n \geq 0 ; k \geq 1},
$$

where $\alpha_{k_{0}}=\alpha+k_{0}$. Proceeding as in the previous case, we obtain that the system $E_{k_{0}}$ forms a basis for $L_{p(\cdot), \rho}$. In this case, the corresponding conjugate problem has the following form

$$
F^{+}\left(e^{i t}\right)+G_{k_{0}}(t) F^{-}\left(e^{i t}\right)=e^{-\alpha_{k_{0}} t} f(t), t \in[-\pi, \pi],
$$

where $G_{k_{0}}(t) \equiv e^{-i\left(2 \alpha+k_{0}\right) t}$. If the inequalities (16), (19) are true, then this problem is uniquely solvable in classes $H_{p(\cdot), \rho}^{+} \times{ }_{-1} H_{p(\cdot), \rho}^{-}$for $\forall f \in L_{p(\cdot), \rho}$. It is obvious that if the conditions (1) are satisfied, then the system $\left\{e^{\mathrm{int}}\right\}_{n \in Z}$ forms a basis for $L_{p(\cdot), \rho}$. If the system $E_{k_{0}}$ forms a basis for $L_{p(\cdot), \rho}$ with some $k_{0} \in Z$, then it is isomorphic to $\left\{e^{\text {int }}\right\}_{n \in Z} \equiv E_{0}$. Let $\mathscr{K}_{\rho}$ denote the space of coefficients of the system $E_{0}$ with regard to $L_{p(\cdot), \rho}$. Consider operator $T: L_{p(\cdot), \rho} \rightarrow L_{p(\cdot), \rho}$ defined by

$$
T f=e^{i \alpha_{k_{0}} t} f^{+}+e^{-i \alpha t} f^{-},
$$

where $f=f^{+}+f^{-}, f^{+} \in H_{p(\cdot), \rho}^{+}, f^{-} \in_{-1} H_{p(\cdot), \rho}^{-}$. It is not difficult to see that

$$
T\left[e^{\mathrm{int}}\right]= \begin{cases}e^{i\left(n+\alpha_{k_{0}}\right) t}, & n \leq 0, \\ e^{-i(n+\alpha) t}, & n>0 .\end{cases}
$$

The unique solvability of the conjugate problem (20) in classes $H_{p(\cdot), \rho}^{+} \times_{-1} H_{p(\cdot), \rho}^{-}$directly implies that the operator $T$ is an isomorphism in $L_{p(\cdot), \rho}$. Consequently, the spaces of coefficients of the systems $E_{k_{0}}$ and $E_{0}$ with regard to $L_{p(\cdot), \rho}$ coincide with each other. Thus, the space of coefficients of the system $E_{k_{0}}$ coincides with the space $\mathscr{K}_{\rho}$. It is absolutely clear that the systems $E_{k_{0}}$ and $E_{0}$ differ from each other by the finite number of elements. Let $C^{k_{0}}$ be a $\left|k_{0}\right|$-dimensional complex space. If $k_{0}=0$, then it is clear that $E \equiv E_{k_{0}}$. If $k_{0}>0$, then $E$ is a part of $E_{k_{0}}$. It is not difficult to see that the following direct decompositions hold:

$$
\begin{aligned}
& \mathscr{K}(E)=C^{k_{0}} \dot{+} \mathscr{K}_{\rho} \quad \text { if } k_{0} \geq 0 ; \\
& \mathscr{K}_{\rho}=C^{k_{0}} \dot{+} \mathscr{K}(E) \text { if } k_{0}<0 .
\end{aligned}
$$

It is absolutely clear that the system $\left\{e^{i(\alpha+k) t}\right\}_{k=0}^{k_{0}-1}$ is linearly independent when $k_{0} \geq 1$. We denote the linear span of this system by $L^{\left(k_{0}\right)}$ and identify it with $C^{k_{0}}$. Consider the direct sum

$$
\hat{L}_{p(\cdot), \rho}=L^{\left(k_{0}\right)} \dot{+} L_{p(\cdot), \rho}=C^{k_{0}} \dot{+} L_{p(\cdot), \rho} .
$$

Obviously, in this case the system $E$ forms a basis for $\hat{L}_{p(\cdot), \rho}$, and, besides, it forms a frame for $\hat{L}_{p(\cdot), \rho}$ with regard to $\mathscr{K}(E)$. When $k_{0}<0$, it is absolutely clear that this system is a frame sequence with regard to $\mathscr{K}(E)$. So we have arrived at the conclusion that the following theorem is true.

Theorem 9. Let $p \in W L, p^{-}>1$, the inequalities (16),(17) be fulfilled, and the set $P_{\pi}$ be defined by (18). Then, the inequalities (21), (22) hold with regard to the space of coefficients of the system (14,) where $k_{0} \in Z$ is defined by (19). When $k_{0}>0$, the system $E$ forms a basis for $\hat{L}_{p(\cdot), \rho}$, where the space $\hat{L}_{p(\cdot), \rho}$ is defined by the direct sum (23). When $k_{0}<0$, the system $E$ is a frame sequence with regard to $\mathscr{K}(E)$.

The author would like to express her deepest gratitude to Professor B.T. Bilalov for his encouragement and valuable guidance throughout this research.

\section{References}

Bilalov, B. T. (1990). Basicity of Some Systems of Exponents, Cosines and Sines. Diff. Uravneniya, 26(1), $10-16$. 
Bilalov, B. T. (1999). On basicity of systems of exponents, cosines and sines in $L_{p}$. Dokl. RAN, 365(1), 7-8.

Bilalov, B. T. (2001). On basicity of some systems of exponents, cosines and sines in $L_{p}$. Dokl. RAN, 379(2), 7-9.

Bilalov, B. T. (2003). Bases of Exponents, Cosines and Sines Which Are Eigenfunctions of Differential Operators. Diff. Uravneniya, 39(5), 1-5.

Bilalov, B. T. (2004). Basis Properties of Some Systems of Exponents, Cosines and Sines. Sibirskiy Matem. Jurnal, 45(2), 264-273.

Bilalov, B. T., \& Guseynov, Z. G. (2012). Basicity of a system of exponents with a piece-wise linear phase in variable spaces. Mediterr. J. Math., 9(3), 48498. http://dx.doi.org/10.1007/s00009-011-0135-7.

Bilalov, B. T., \& Guseinov, Z. G. (2011). A criterion for the basis property of perturbed exponential systems in Lebesgue spaces with variable exponent. Doklady Mathematics, 83(1), 93-96.

Christensen, O. (2003). An Introduction to Frames and Riesz bases. Birkhaeuser, Boston, Basel, Berlin.

Danilyuk, I. I. (1975). Non-regular boundary value problems in the plane, M., Nauka, 256.

Duffin, R. J., \& Schaeffer, A. C. (1952). A class of no harmonic Fourier series. Trans. Amer. Math. Soc., 72(341), 341-366.

Heil, C. (2011). A Basis theory primer. Springer, 536.

Kokilashvili, V., \& Paatashvili, V. (2006). On Hardy classes of analytic functions with a variable exponent. Proc. Razmadze Math.Inc., 142, 134-137.

Kokilashvili, V., \& Samko, S. (2003). Singular integrals in weighted Lebesgue spaces with variable exponent. Georgian Math. J., 10(1), 145-156.

Kovacik, O., \& Rakosnik, J. (1991). On spaces $L^{p(\cdot)}$ and $W^{k, p(\cdot)}$. Czechoclovak Math. I., 41(116), 592-618.

Kusis, P. (1984). Introduction to the Theory of Spaces $H^{p}$. M., «Mir», 364.

Mamedova, Z. V. (2012). On basis properties of degenerate exponential system. Applied Mathematics, 3, 1963-1966. http://dx.doi.org/10.4236/am.2012.312269

Moiseev, E. I. (1984). On basicity of systems of sines and cosines. DAN SSSR, 275(4), 794-798.

Moiseev, E. I. (1987). On basicity of a system of sines. Diff. Uravneniya, 23(1), 177-179.

Moiseev, E. I. (1998). On Basicity of Systems of Sines and Cosines in Weighted Space. Diff. uravneniya, 34(1), 40-44.

Moiseev, E. I. (1999). Basicity of a System of Eigenfunctions of a Differential Operator in a Weighted Space. Diff. Uravneniya, 35(2), 200-205.

Paley, R., \& Wiener, N. (1934). Fourier Transforms in the Complex Domain. Amer. Math. Soc. Colloq. Publ., 19 (Amer. Math. Soc., Providence, RI).

Privalov, I. I. (1950). Boundary Properties of Analytic Functions. M-L, Gostekhizdat, 336.

Sadigova, S. R., \& Mamedova, Z. V. (2013). Frames from cosines with the degenerate coefficients. American Journal of Applied Mathematics and Statistics, 1(3), 36-40.

Sedletskii, A. M. (2005). Classes of Analytic Fourier Transforms and Exponential Approximations. Moscow, Fizmatlit, 504.

Sharapudinov, I. I. (2007). Some problems of approximation theory in spaces $L^{p(x)}(\mathrm{E})$. Anal.Math., 33(2), 135-153.

Xianling, F., \& Dun, Z. (2001). On the spaces $L^{p(x)}(\Omega)$ and $W^{m, p(\cdot)}(\Omega)$. Journal of Math. Anal. and Appl., 263, 424-446

Young, R. M. (1980). An Introduction to Nonharmonic Fourier series. Springer, 246.

\section{Copyrights}

Copyright for this article is retained by the author(s), with first publication rights granted to the journal.

This is an open-access article distributed under the terms and conditions of the Creative Commons Attribution license (http://creativecommons.org/licenses/by/3.0/). 